HNO 2022 $\cdot 70: 44-50$

https://doi.org/10.1007/s00106-021-01029-w

Angenommen: 28. Januar 2021

Online publiziert: 9. April 2021

๑) Der/die Autor(en) 2021

Matti Sievert ${ }^{1} \mathbb{D}$ - Miguel Goncalves ${ }^{1}$ - Benedicta Binder ${ }^{1}$ Sarina K. Mueller' . Robin Rupp ${ }^{1}$. Michael Koch ${ }^{1}$ - Stephan Dürr' ${ }^{1}$ Maximilian Traxdorf' . Markus Hecht ${ }^{2} \cdot$ Heinrich Iro' ${ }^{1}$ Antoniu-Oreste Gostian'

${ }^{1}$ Hals-Nasen-Ohrenklinik, Kopf- und Hals-Chirurgie, Universitätskliniken Erlangen, Friedrich-AlexanderUniversität Erlangen-Nürnberg, Erlangen, Deutschland

${ }^{2}$ Strahlenklinik, Universitätskliniken Erlangen, Friedrich-Alexander-Universität Erlangen-Nürnberg, Erlangen, Deutschland

\title{
Salvage-Laryngektomie nach primärer Radio- und Radiochemotherapie
}

\section{Eine retrospektive Fallzusammenfassung}

\section{Zusatzmaterial online \\ Die Online-Version dieses Beitrags (https://doi.org/10.1007/s00106-021- 01029-w) enthält eine Übersicht über die Patientenkohorte. Beitrag und Zusatzmaterial stehen Ihnen auf www.springermedizin. de zur Verfügung. Bitte geben Sie dort den Beitragstitel in die Suche ein, das Zusatzmaterial finden Sie beim Beitrag unter „Ergänzende Inhalte“.

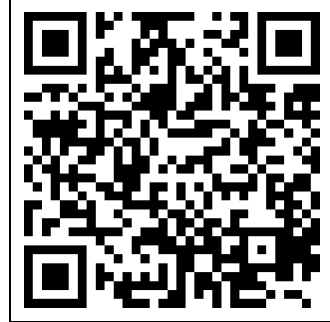

Die Salvage-Laryngektomie ist eine mögliche Therapieoption in der Behandlung des rezidivierenden und residuellen Larynxkarzinoms nach erfolgter $\mathrm{Ra}$ dio- oder Radiochemotherapie. Unter ständiger Konkurrenz und der rapiden Weiterentwicklung nichtoperativer Behandlungsmethoden des Kopf-HalsMalignoms in den letzten Jahren ist die Erfassung der onkologischen Ergebnisse und der Komplikationen der SalvageChirurgie essenziell für die klinische Entscheidungsfindung.

Die englische Version dieses Beitrags ist unter https://doi.org/10.1007/s00106-021-01030-3 zufinden.

\section{Hintergrund}

Trotz zunehmender Tendenz zu primär organerhaltenden Ansätzen bei lokal fortgeschrittenen Plattenepithelkarzinomen des Larynx und des Hypopharynx kommt der „Rettungschirurgie“ (Salvage-Chirurgie) weiterhin eine große Bedeutung zu. Abhängig von der Lokalisation und dem Stadium des Primärtumors im Kopf-Hals-Bereich liegen die Rezidivraten nach primär organerhaltender Therapie bei 25-50\% [1]. In der Behandlung des lokoregionären Rezidivs ist eine wiederholte Strahlentherapie häufig ausgeschlossen, sodass mit der Rettungschirurgie effektive onkologische Ergebnisse erzielt werden können [2]. Die Wundheilung nach Strahlen- und Chemotherapie ist durch die Fibrosierung des Gewebes und eine Verminderung der Perfusion beeinträchtigt [3, 4]. Daraus ergibt sich eine deutlich erhöhte Komplikationsrate von bis $\mathrm{zu} 60 \%$, verbunden mit deutlich gesteigerter Mortalität und Morbidität sowie einer signifikant beeinträchtigten Lebensqualität [5]. Die Inkaufnahme der bekannten Nachteile und Risiken der Rettungschirurgie kann nur durch ein verbessertes Überleben gerechtfertigt werden. Als Alternative zur Operation stehen nach Versagen der Erstlinientherapie heutzutage neben der palliativen Chemotherapie und der Wiederbestrahlung auch die Immuntherapie zur Verfügung [6]. Es gibt zahlreiche klinische Studien, die die Rolle von Checkpointinhibitoren sowohl als Einzelsubstanz als auch in Kombination mit etablierten Behandlungsmethoden untersuchen. Der aktuelle Paradigmenwechsel in der Therapie des rekurrenten fortgeschrittenen Larynxkarzinoms stellt erneut die Wertigkeit der Rettungschirurgie auf den Prüfstand. Das Ziel der vorliegenden Arbeit war die Bestimmung der Überlebensrate sowie die Bewertung präoperativer prognostischer Faktoren für das allgemeine und krankheitsfreie Überleben einer Rettungschirurgie von Residualtumoren oder Rezidiven anhand der an der Klinik der Autor(inn)en behandelten Patienten. Sekundäre Ziele umfassten die Auswertung chirurgischer und allgemeiner postoperativer Komplikationen und die Dauer der Sondenernährung.

\section{Studiendesign und Unter- suchungsmethoden}

Es handelt sich um eine retrospektive Kohortenstudie der Universitätsklinik Erlangen (Abteilung für Hals-Nasen-Ohrenheilkunde, Kopf- und Hals-Chirurgie). Die Studie wurde von der zuständigen Ethikkommission genehmigt (Votum 246_20 Bc) und in Einklang mit der Deklaration von Helsinki durchgeführt. 


\begin{tabular}{|c|c|c|}
\hline Geschlecht & \multicolumn{2}{|l|}{$n(\%)$} \\
\hline Männlich & \multicolumn{2}{|l|}{$27(81,8)$} \\
\hline Weiblich & \multicolumn{2}{|l|}{$6(18,2)$} \\
\hline Variable & Initial & Salvage \\
\hline Alter & $M W \pm S D$ & $M W \pm S D$ \\
\hline Jahre & $61,2 \pm 10$ & $63,9 \pm 10$ \\
\hline T-Stadium & $n(\%)$ & $n(\%)$ \\
\hline $\mathrm{T} 1$ & $1(3,0)$ & $5(15,1)$ \\
\hline $\mathrm{T} 2$ & $9(27,3)$ & $4(12,1)$ \\
\hline T3 & $12(36,4)$ & $9(27,3)$ \\
\hline T4a & $11(33,3)$ & $12(36,4)$ \\
\hline $\mathrm{T} 4 \mathrm{~b}$ & - & $3(9,1)$ \\
\hline N-Stadium & $n(\%)$ & $n=17(\%)^{a}$ \\
\hline No & $16(48,5)$ & $15(88,2)$ \\
\hline N1 & $4(12,1)$ & - \\
\hline $\mathrm{N} 2 \mathrm{~b}$ & $7(21,2)$ & - \\
\hline $\mathrm{N} 2 \mathrm{C}$ & $6(18,2)$ & - \\
\hline $\mathrm{N} 3 \mathrm{~b}$ & - & $2(11,8)$ \\
\hline UICC & $n(\%)$ & $n(\%)$ \\
\hline I & $1(3,0)$ & $5(15,2)$ \\
\hline II & $4(12,2)$ & $4(12,1)$ \\
\hline III & $11(33,3)$ & $7(21,1)$ \\
\hline IVa & $17(51,5)$ & $12(36,4)$ \\
\hline $\mathrm{IVb}$ & - & $5(15,2)$ \\
\hline Grading & $n(\%)$ & $n=31(\%)$ \\
\hline G1 & - & $1(3,2)$ \\
\hline G2 & $15(45,5)$ & $10(32,3)$ \\
\hline G3 & $18(54,5)$ & $20(64,5)$ \\
\hline$E C O G$ & $n=32(\%)$ & $n(\%)$ \\
\hline ECOG 0 & $9(28,1)$ & $4(12,1)$ \\
\hline ECOG 1 & $13(40,7)$ & $9(27,3)$ \\
\hline ECOG 2 & $8(25,0)$ & $15(45,5)$ \\
\hline ECOG 3 & $1(3,1)$ & $4(12,1)$ \\
\hline ECOG 4 & $1(3,1)$ & $1(3,0)$ \\
\hline $\begin{array}{l}\text { UICC Union Ir } \\
\text { Status, MW N } \\
{ }^{2} \text { Bei } 16 \text { ist kei }\end{array}$ & $\begin{array}{l}\text { e le Cancer, } E C \\
\text { ardabweichur } \\
\text { erfolgt }\end{array}$ & tive Oncology Group Performance \\
\hline
\end{tabular}

\section{Studiendesign}

Die Analyse erfolgte mittels retrospektiver Auswertung von Patientenakten aus der Tumordatenbank der Klinik. Die ausgewählten Patienten haben wegen eines Larynxkarzinoms im Zeitraum von März 2001 bis Oktober 2019 in dem akademischen Tumorzentrum der Autor(inn)en eine totale oder partielle Laryngektomie erhalten. Einschlusskriterien waren ein Tumorresiduum oder ein Lokalrezidiv nach primär organerhaltender nichtchirurgischer Therapie. Als Residuum wurde
Exstirpation des Larynx bzw. Teillaryngektomie mit oder ohne begleitender Pharyngektomie wurden die Defekte je nach Größe primär verschlossen oder mit regionalen oder freien mikrovaskulären Lappenplastiken rekonstruiert. Die Anlage einer perkutanen endoskopischen Gastrostomie (PEG) erfolgte als prophylaktische Maßnahme. Eine Gastrografin-Ösophagographie wurde nach 10 Tagen postoperativ durchgeführt. Die Autor(inn)en dokumentierten die onkologischen Parameter (TNM, R-Status), postoperative Komplikationen und den Status gemäß ECOG (Eastern Cooperative Oncology Group). Die Klassifikation des Erkrankungsstadiums erfolgte anhand der 8. Version der UICC (Union Internationale Contre le Cancer [7]).

\section{Zielparameter}

Primäre Endpunkte der Studie waren die onkologischen Ergebnisse mit der Rate an lokalen und regionären Rezidiven und Fernmetastasen sowie das krankheitsfreie Überleben und das Gesamtüberleben. Die Überlebenszeit wurde vom Tag der Operation bis zum Todestag aufgrund einer beliebigen Ursache (Gesamtüberleben), dem Auftreten eines Rezidivs (krankheitsfreies Überleben), oder des Datums, an dem der Patient zuletzt als lebend (Gesamtüberleben und krankheitsfreies Überleben) oder als nicht krankheitsbedingt tot (krankheitsfreies Überleben) bekannt war, definiert. Die Autor(inn)en zensierten Patienten, die zum Zeitpunkt der Auswertung noch am Leben waren. Sekundäre Endpunkte stellten die Rate an postoperativen Komplikationen, die Dauer der Sondenernährung über die PEG und die Art der Ernährung bei der letzten Nachsorge dar.

\section{Statistische Auswertung}

Die metrischen Parameter werden mit dem Mittelwert und der Standardabweichung (SD) angegeben. Die Häufigkeiten der Variablen werden in absoluten und relativen Werten $(n ; \%)$ dargestellt. Die Überlebenskurven wurden mithilfe der Kaplan-Meier-Schätzung erstellt und anhand des Log-Rank-Tests verglichen. As- 
HNO 2022 · 70:44-50 https://doi.org/10.1007/s00106-021-01029-w

(c) Der/die Autor(en) 2021

M. Sievert · M. Goncalves · B. Binder · S. K. Mueller · R. Rupp · M. Koch · S. Dürr · M. Traxdorf · M. Hecht · H. Iro · A.-O. Gostian

\section{Salvage-Laryngektomie nach primärer Radio- und Radiochemotherapie. Eine retrospektive Fallzusammenfassung}

\section{Zusammenfassung}

Hintergrund. Das rezidivierende und residuelle Larynxkarzinom nach organerhaltender Radio- bzw. Radiochemotherapie ist mit einer schlechten Prognose verbunden. Die SalvageOperation stellt in diesen Fällen die wichtigste therapeutische Option dar.

Ziel der Arbeit. Erfasst wurden die Rate an Rezidiv- und Residualtumoren sowie die Überlebensraten und die Komplikationsrate nach Salvage-Chirurgie des Kehlkopfs an dem akademischen Tumorzentrum der Autor(inn)en.

Material und Methoden. Retrospektiv wurden alle Patienten untersucht, bei denen zwischen 2001 und 2019 eine SalvageOperation aufgrund eines Tumorresiduums oder Rezidivs nach primärer nichtchirurgischer Therapie erfolgt war.
Ergebnisse. Es wurden 33 Salvage-Operationen durchgeführt. Die Defektrekonstruktion erfolgte in $30,3 \%$ der Fälle $(n=10)$ mittels freier und in $15,2 \%(n=5)$ mittels regionaler Lappenplastik. Ein Patient hat sowohl eine freie als auch gleichzeitig eine gestielte Lappenplastik erhalten. Das Gesamtüberleben nach einem, 2 und 5 Jahren betrug $68,7 \%$; $47,9 \%$ bzw. $24,2 \%$, das krankheitsfreie Überleben $81,6 \% ; 47,8 \%$ bzw. $24,2 \%$ bei insgesamt $48,5 \%(n=16)$ postoperativen Tumorrezidiven. Das krankheitsfreie Überleben war signifikant kürzer bei Tumorausdehnung im bzw. auf den Hypopharynx $(p=0,041)$. Postoperativ entwickelten $72,7 \%$ der Patienten eine pharyngokutane Fistel unabhängig von einer simultanen Defektrekonstruktion. Nur 24\% der der aufgetretenen Fisteln mussten operativ therapiert werden. Der Krankenhausaufenthalt betrug $28,0 \pm 16,1$ Tage.

Schlussfolgerung. Die Salvage-Laryngektomie ist mit vielen, aber beherrschbaren Komplikationen und einer hohen Morbidität verbunden. In Anbetracht der behandelten fortgeschrittenen Tumorkategorien und der Gesamtsituation des Patienten sind respektable onkologische Ergebnisse zu erreichen.

\section{Schlüsselwörter}

Rezidiv · Salvage-Therapie · Larynxkarzinom $\cdot$ Therapie-Outcome $\cdot$ Postoperative Komplikationen

\section{Salvage laryngectomy after primary radio- and radiochemotherapy. A retrospective study. German version}

\section{Abstract}

Background. Recurrent and residual laryngeal cancer after organ-preserving radio- or radiochemotherapy is associated with a poor prognosis. Salvage surgery is the most important therapeutic option in these cases. Objective. The study assessed rates of recurrence and residual tumor as well as survival and complication rates after salvage laryngectomy at the authors' academic cancer center.

Materials and methods. A retrospective examination of all patients receiving laryngectomy between 2001 and 2019 due to tumor residuals or recurrence after primary radioand radiochemotherapy was conducted.
Results. A total of 33 salvage procedures were performed. Defect reconstruction was performed by free flap surgery in $30.3 \%$ $(n=10)$ and regional flap surgery in $15.2 \%$ $(n=5)$. One patient received regional flap surgery and free flap surgery simultaneously. Overall survival after 1,2 , and 5 years was 68.7, 47.9 , and $24.2 \%$, and disease-free survival was $81.6,47.8$, and $24.2 \%$, respectively, with $48.5 \%(n=16)$ postoperative tumor recurrences overall. Disease-free survival was significantly shorter for tumor extension into or onto the hypopharynx $(p=0.041)$. Postoperatively, $72.7 \%$ of patients developed a pharyngocutaneous fistula, of which $24.2 \%$ required surgical treatment. The hospital stay was $28.0 \pm 16.1$ days.

Conclusion. Salvage laryngectomy is associated with a high rate of treatable complications and high morbidity. Nevertheless, considering the advanced tumor stages treated, it allows for respectable oncological results.

\section{Keywords}

Recurrence - Salvage therapy · Laryngeal neoplasms - Treatment outcome - Postoperative complications soziationen zwischen einzelnen anatomischen Lokalisationen mit dem Gesamtüberleben sowie dem krankheitsfreien Überleben wurden an univariaten CoxModellen getestet. Der Zusammenhang zwischen nominalen Variablen wurde mit dem $\chi^{2}$-Test geprüft. Ein $p$-Wert $<0,05$ wurde als statistisch signifikant angesehen. Für die statistische Auswertung verwendeten die Autor(inn)en IBM SPSS Statistics, Version 25.0 (Fa. IBM Corporation, Armonk/NY, USA).

\section{Ergebnisse}

\section{Merkmale des Patientenkollektivs}

Insgesamt wurden in dem angegebenen Zeitraum an dem Zentrum der Autor(inn)en 1327 Plattenepithelkarzinome des Larynx diagnostiziert. Bei 1134 Patienten erfolgte eine primär operative und bei 193 Patienten eine primär organerhaltende Therapie. Darunter erfüllten 33 Patienten die Einschlusskri- terien (6 w., 27 m.; mittleres Alter: $63,9 \pm 10$ Jahre, $\bullet$ Tab. 1). Die Erstlinientherapie erfolgte in 8 Fällen $(24,2 \%) \mathrm{mit}$ einer intensitätsmodulierten Radiotherapie (bis $67,3 \pm 3,9$ Gy Gesamtdosis). In 25 Fällen $(75,8 \%)$ wurde eine simultane Radiochemotherapie (bis $69,4 \pm 3,4 \mathrm{~Gy}$ Gesamtdosis; Cisplatin, 5-Fluorouracil, Docetaxel). Bei 8 Patienten war eine Induktionstherapie vorausgegangen.

Im Fall eines Tumorresiduums $(n=12$; $36,4 \%)$ erfolgte die Salvage-Operation 


\begin{tabular}{|c|c|c|}
\hline Primäre Therapie & $n$ & $\%$ \\
\hline Radiotherapie & 8 & 24,2 \\
\hline Radiochemotherapie ${ }^{a}$ & 25 & 75,8 \\
\hline Induktion $^{b}$ & 8 & 24,2 \\
\hline Bestrahlungsdosis (Gy) & MW & SD \\
\hline Tumorregion & 68,9 & 3,6 \\
\hline Lymphabflussgebiet & 58,2 & 6,2 \\
\hline Zeitspanne (Bestrahlung - Op.) & MW & SD \\
\hline Monate & 18,6 & 16,9 \\
\hline Indikation zur Salvage-Laryngektomie & $n$ & $\%$ \\
\hline Residuum & 12 & 36,4 \\
\hline Rezidiv & 20 & 60,6 \\
\hline Zweitmalignom & 1 & 3,0 \\
\hline Resektionsstatus & $n$ & $\%$ \\
\hline RO & 30 & 90,9 \\
\hline R1 & 3 & 9,1 \\
\hline Pharynxrekonstruktion & $n$ & $\%$ \\
\hline Primäre Pharynxnaht & 19 & 57,6 \\
\hline Gestielter Lappen ${ }^{c, d}$ & 5 & 15,2 \\
\hline Freier Lappene & 10 & 30,3 \\
\hline Neck-Dissection & $n$ & $\%$ \\
\hline Unilateral & 3 & 9,1 \\
\hline Bilateral & 14 & 42,4 \\
\hline \multicolumn{3}{|c|}{$\begin{array}{l}\text { MW Mittelwert, SD Standardabweichung } \\
{ }^{a} \text { Simultane Radiochemotherapie } \\
\text { bInduktionschemotherapie (Cisplatin, 5-Fluorouracil [5-FU], Docetaxel) } \\
\text { 'Patient Nr. } 15 \text { wurde zur Rekonstruktion des Pharynx mit einem freien und einem gestielten Transplan- } \\
\text { tat versorgt } \\
{ }^{\mathrm{d} D} \text { Davon } 3 \text { Deltopektorallappen und } 2 \text { Pektoralis-major-Lappen } \\
\text { eDavon } 4 \text { anterolaterale Oberschenkellappen und } 6 \text { Radialislappen }\end{array}$} \\
\hline
\end{tabular}

nach durchschnittlich 7,2 $\pm 3,8$ Monaten. Patienten mit Lokalrezidiven ( $n=20 ; 60,6 \%)$ wurden durchschnittlich $25,4 \pm 18,1$ Monate nach Erstdiagnose operiert. Ein Patient (3\%) entwickelte nach einem tumorfreien Intervall von 27 Jahren ein Rezidiv. In • Tab. 1 sind die Patienten- und Therapiecharakteristika zum Zeitpunkt der Erstdiagnose und bei der Salvage-Operation aufgeführt. Eine Übersicht über die Patientenkohorte ist im elektronischen Zusatzmaterial online zu finden.

\section{Salvage-Chirurgie}

Die insgesamt 33 Salvage-Operationen erfolgten mittels kompletter $(n=31)$ oder partieller $(n=2)$ Laryngektomie. Tumorfreie Schnittränder (R0) konnten insgesamt in 30 Fällen $(90,9 \%)$ erreicht werden. Bei 3 Patienten $(9,1 \%)$ wurde eine R1-Situation vorgefunden. In 2 Fällen in 2 Fällen $(6,1 \%)$ histopathologisch bestätigt werden. Die pathologische Reklassifizierung ergab: $5(15,1 \%)$ rpT1, $4(12,1 \%) \quad r p T 2,9(27,3 \%) \quad r p T 3,15$ (45,5\%) rpT4 (• Tab. 1). In 19 Fällen (57,6\%) erfolgte die Pharynxrekonstruktion mittels primärer Naht. Aufgrund größerer Resektionsdefekte wurde bei 10 Patienten (30,3\%) eine freie mikrovas- kuläre Lappenplastik und bei 5 Patienten $(15,2 \%)$ eine regionale Lappenplastik mittels myokutanem Pektoralis-majorLappen $(n=2)$ oder fasziokutanem Deltopektorallappen $(n=3)$ durchgeführt (- Tab. 2). Davon erhielt ein Patient sowohl eine freie als auch eine gestielte Lappenplastik. Bei 28 Patienten (84,8\%) erfolgte im Rahmen der Therapie eine PEG-Versorgung (• Tab. 3). Um eine Stimmrehabilitation $\mathrm{zu}$ erreichen, wurde bei 6 Patienten (18,2\%) eine primäre und bei einem Patienten (3,0\%) eine sekundäre tracheoösophageale Punktion mit Anlage einer Stimmprothese (Provox 1, 2 und Vega; Fa. Atos Medical, Malmö, Schweden) durchgeführt.

\section{Onkologische Ergebnisse}

Insgesamt wiesen 16 der 33 Patienten (48,5\%) ein Tumorrezidiv nach der Salvage-Operation auf. Bei 9 Patienten $(27,3 \%)$ wurde ein Lokalrezidiv nach durchschnittlich $83,4 \pm 87,8$ Wochen und bei 3 Patienten $(9,1 \%)$ ein regionäres, zervikales Rezidiv nach 159,6 $\pm 121,2$ Wochen festgestellt. Fernmetastasen wurden bei 11 Patienten $(33,3 \%)$ nach durchschnittlich 76,36 $\pm 80,6$ Wochen diagnostiziert. Die Ein-, 2- und 5-Jahres-Gesamtüberlebensrate betrug 68,7\%, 47,9\% bzw. 24,2\% mit einer medianen Überlebenszeit von 11 Monaten (0-206 Monate). Das krankheitsfreie Überleben nach einem, 2 und 5 Jahren betrug 81,6\%, 47,8\% bzw. 24,2\% (• Abb. 1). In der univariaten Regressionsanalyse erwies sich die Tumormanifestation im Hypopharynx $(p=0,033)$ als signifikanter negativer Prädiktor für das Gesamtüberleben. Das krankheitsfreie Überleben war signifikant reduziert im Fall einer Tumormanifestation im Hypopharynx $(p=0,041)$.

\section{Postoperativer Verlauf}

Eine Übersicht über alle chirurgischen und medizinischen postoperativen Komplikationen gibt - Tab. 3. Komplikationen traten insgesamt bei 25 Patienten $(75,7 \%)$ auf. Die pharyngokutane Fistel war mit $72,7 \%(n=24)$ die häufigste Komplikation. In 16 Fällen (48,5\%) verschloss sich die Fistel nach rein konser- 

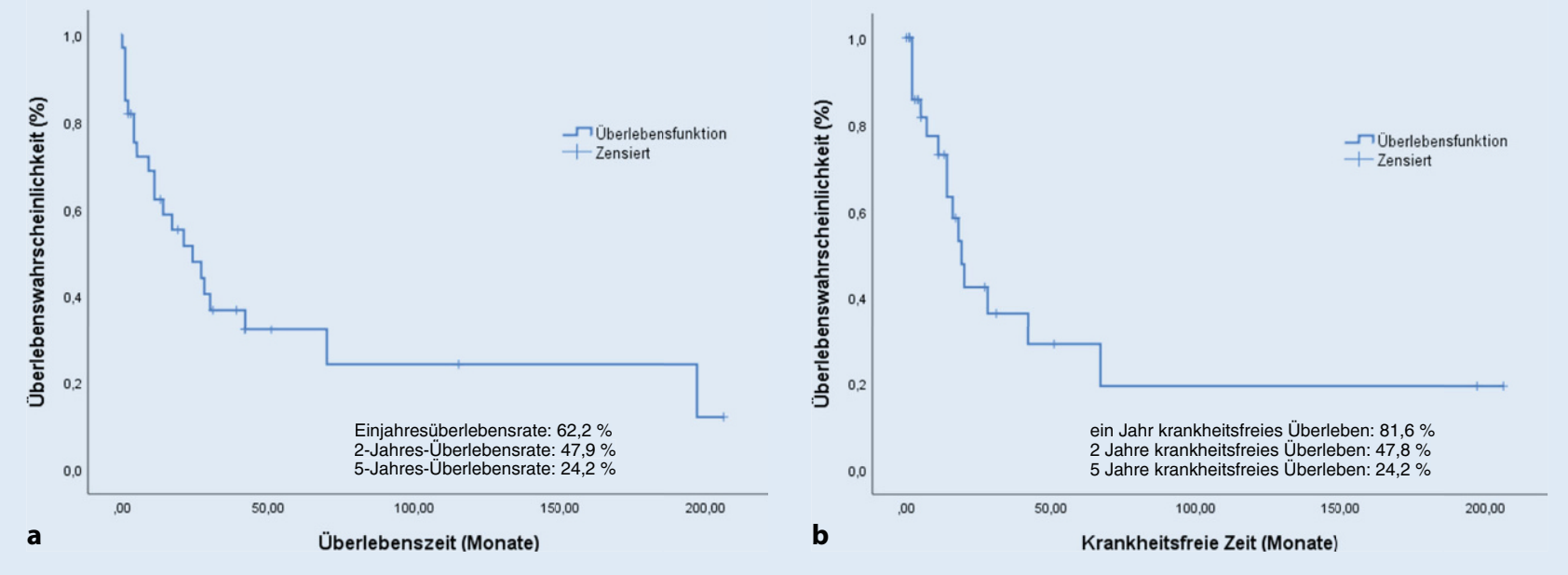

Abb. 1 \ Gesamtüberleben (a) und krankheitsfreies Überleben (b)

vativer Behandlung nach durchschnittlich 46,9 $\pm 35,6$ Tagen. Bei 8 Patienten $(24,2 \%)$ wurde ein operativer Fistelverschluss mittels Pektoralis-major- oder Deltopektorallappen erforderlich. Die Autor(inn)en konnten keinen Zusammenhang zwischen Patienten mit und ohne primäre Lappenrekonstruktion in Bezug auf die Fistelrate feststellen $(p=0,618)$. Der Patient mit sekundärer Anlage einer Provox-Prothese entwickelte eine ausgedehnte ösophagotracheale Fistel. Die Patienten waren durchschnittlich 28,0 $\pm 16,1$ in stationärer Betreuung, davon $6,0 \pm 5,8$ Tage auf der Intensivstation. Bei der letzten Nachsorgeuntersuchung nach durchschnittlich $34,0 \pm 50,8$ Monaten ernährten sich 16 Patienten (50,0 \%) vollständig oral. Die PEG wurde bei diesen Patienten nach durchschnittlich 39,7 $\pm 20,8$ Wochen entfernt. Bei der letzten Vorstellung waren 5 Patienten $(15,6 \%)$ teilweise und 11 Patienten $(34,4 \%)$ vollständig auf die PEG angewiesen (• Tab. 3).

\section{Diskussion}

In der vorliegenden Arbeit wurde der Verlauf von Patienten nach Rettungschirurgie durch eine totale Laryngektomie oder Teillaryngektomie nach primär nichtchirurgischer Therapie untersucht. Die vorliegenden Ergebnisse zeigen ein 2- und ein 5-Jahres-Gesamtüberleben von 47,9 bzw. $24,2 \%$ (• Abb. 1) und ein krankheitsfreies Überleben von 47,8 bzw. $24,2 \%$. Insgesamt wiesen $48,5 \%$ der Patienten postoperativ ein Tumorrezidiv auf. Die Autor(inn)en stellten eine signifikant schlechtere 5-Jahres-Überlebensrate fest, wenn eine hypopharyngeale Beteiligung vorlag $(28,8$ vs. $10,9 \% ; p=0,041)$. Die Rettungschirurgie war mit einer hohen Komplikationsrate verbunden. Dabei war die pharyngokutane Fistel die häufigste Komplikation, die zumeist lediglich einer konventionellen Therapie bedurfte. Die Hälfte der behandelten Patienten konnte sich postoperativ komplett oral ernähren.

Allgemein liegt die Rate der lokoregionären Rezidive nach organerhaltender Therapie bei $30-50 \%[1,8]$. Besteht nach primärer Radiochemotherapie ein Tumorresiduum oder ein lokoregionäres Rezidiv, sollte die Möglichkeit der Salvage-Chirurgie geprüft werden. Sie stellt nach Versagen der primär nichtchirurgischen Therapie weiterhin eine kurative Therapieform dar, sofern eine vollständige Resektion mit negativen Rändern präoperativ erreichbar erscheint. Über $70 \%$ der Patienten mit einem Residuum oder Rezidiven nach Radiochemotherapie weisen lokal fortgeschrittene Tumoren der Kategorien T3 und T4 auf [9]. Dies können die Autor(inn)en mit 27,3 \% der Patienten in einem frühen (UICC I und II) und $72,7 \%$ der Patienten in einem lokal fortgeschrittenen Tumorstadium (UICC III und IV) bestätigen. In
Anbetracht des Untersuchungszeitraums und der darin begründeten Entwicklung der Bestrahlungstechnik sind die von den Autor(inn)en mittels Rettungschirurgie behandelten Patienten mit den Angaben von Putten et al. [5] für die Untersuchungsjahre 1990-2007 vergleichbar. Damit bleibt die Rettungschirurgie für den onkologischen Chirurgen weiterhin eine unverändert anspruchsvolle und komplikationsbehaftete chirurgische Therapiemöglichkeit.

Die onkologischen Ergebnisse sind therapieübergreifend insgesamt unbefriedigend. Für rein laryngeale Karzinome wird zusammengefasst ein Gesamtüberleben von $48-49 \%$ und mit Beteiligung des Hypopharynx von 17-26\% angegeben $[1,10]$. Alternativ kann eine erneute Radio- oder Radiochemotherapie in kurativer Absicht angeboten werden. Eine umfassende Metaanalyse zu den Ergebnissen der Wiederbestrahlung wurde von Grün et al. kürzlich veröffentlicht. Die Kollegen berichten über eine 2- und 5-Jahres-Gesamtüberlebensrate von $47-57 \%$ und $23 \%$ nach intensitätsmodulierter Radiotherapie mit begleitender Chemotherapie [11]. Beachtlich sind die damit verbundenen, teilweise gravierenden Nebenwirkungen mit schwerwiegenden Akutreaktionen in bis zu 73\%. Akute lebensbedrohliche Komplikationen wurden in bis zu $11 \%$ der Fälle beschrieben [11, 12]. Die alleinige palliative Therapie führte 


\begin{tabular}{|c|c|c|}
\hline Chirurgische Komplikationen & $n(\%)$ & Dauer in Tagen $(M W \pm S D)$ \\
\hline Fistel ohne chirurg. Revision & $16(48,5)$ & $46,9 \pm 35,6$ \\
\hline Fistel mit chirurg. Revision & $8(24,2)$ & $201,0 \pm 170,9$ \\
\hline Ösophagotracheale Fistel & $1(3,0)$ & - \\
\hline Wundheilungsstörungen & $14(42,4)$ & - \\
\hline Medizinische Komplikationen & $n(\%)$ & \multirow[t]{3}{*}{-} \\
\hline Lungenarterienembolie & $1(3,0)$ & \\
\hline Schlaganfall & $2(6,1)$ & \\
\hline \multirow[t]{2}{*}{ Dauer des stationären Aufenthalts } & \multirow[t]{2}{*}{-} & Dauer in Tagen $(M W \pm S D)$ \\
\hline & & $28,0 \pm 16,1$ \\
\hline \multirow[t]{2}{*}{ Dauer des Aufenthalts auf Intensivstation } & \multirow[t]{2}{*}{-} & Dauer in Tagen $(M W \pm S D)$ \\
\hline & & $6,0 \pm 5,8$ \\
\hline PEG-Versorgung & $n(\%)$ & Dauer in Wochen $(M W \pm S D)$ \\
\hline Gesamt & $28(84,8)$ & \multirow[t]{4}{*}{$39,7 \pm 20,8$} \\
\hline Vor Salvage-Operation & $19(57,6)$ & \\
\hline Bei Salvage-Operation & $8(24,2)$ & \\
\hline Nach Salvage-Operation & $1(3,0)$ & \\
\hline Ernährung bei letzter Vorstellung & $n=32(\%)$ & \multirow[t]{4}{*}{-} \\
\hline Komplett über PEG-Sonde & $11(34,4)$ & \\
\hline Teilweise über PEG-Sonde & $5(15,6)$ & \\
\hline Normale oder weiche Kost (d. h. PEG entfernt) & $16(50,0)$ & \\
\hline \multicolumn{3}{|c|}{ PEG perkutane endoskopische Gastrostomie, MW Mittelwert, SD Standardabweichung } \\
\hline
\end{tabular}

Kowalski et al. zufolge an 797 Patienten mit rezidivierendem Kopf-HalsMalignomen $\mathrm{zu}$ einem medianen Überleben von nur 3,8 Monaten und ist bei möglicher Operabilität zurückhaltend zu empfehlen [13]. Eine erschwerte intraoperative Präparation durch narbige postradiogene Veränderungen und die Minderdurchblutung des Gewebes sind charakteristisch für eine Salvage-Operation [2]. Dies bedingt die deutlich erhöhten postoperativen Komplikationsraten mit Wundheilungsstörungen, Lymphödemen und v.a. der Ausbildung von pharyngokutanen Fisteln, die die häufigste chirurgische Komplikation darstellen [4].

Die konservative Therapie steht in solchen Fällen im Vordergrund, sodass nur bei rund einem Drittel aller Fisteln eine operative Versorgung notwendig ist [4, $14,15]$. Insbesondere Patienten mit einer revisionsbedürftigen Fistel zeigten einen prolongierten Heilungsverlauf mit deutlicher Einschränkung der Lebensqualität [15]. Die Rekonstruktion des Pharynx mit frischem Gewebe, bespielweise durch die Präparation eines Pektoralis-majorLappens, hat einen protektiven Effekt auf die Fistelrate [15]. In Anbetracht der zu
Datenerhebung, der wirkungsvolle Entwicklungen der Radioonkologie umfasst. Dennoch sind die Charakteristika der zu behandelnden Patienten mit denen aus den 1990er- und ersten 2000er-Jahren vergleichbar.

Die Rettungschirurgie des Kehlkopfkarzinoms ist weiterhin eine Therapiemöglichkeit, die auch heutzutage noch ihren Stellenwert gegenüber alternativen Optionen der Wiederbestrahlung hat und in diesen anspruchsvollen Situationen mit dem Patienten ausführlich erörtert werden sollte.

\section{Fazit für die Praxis}

- Die Salvage-Laryngektomie bleibt nach Organerhaltungsprotokollen die derzeit beste Therapieoption von Residuen und Rezidiven in kurativer Intention und sollte als Therapieoption in Betracht gezogen werden.

- Insbesondere Pateinten mit kleinen, rein laryngealen Tumoren ohne zervikale Metastasierung profitieren von einer Salvage-Laryngektomie. Salvage-Chirurgie und zum Erhalt einer akzeptablen Lebensqualität und Ernährung trägt auch die Anlage einer PEG bei [16, 17]. Zwei Drittel der Patienten waren bei der letzten Vorstellung, nach durchschnittlich 25 Monaten, nur teilweise oder gar nicht auf die Sondenernährung angewiesen.

Aufgrund der Problematik eines präoperativen „understaging“, bedingt durch postradiogene Ödeme und narbige Veränderungen, sowie der häufig multizentrischen Tumorherde der Rezidive ist die totale Laryngektomie weiterhin das präferierte Verfahren in der SalvageChirurgie des Larynxkarzinoms [10, 18, 19]. Die Ergebnisse dieser seltenen und anspruchsvollen Therapie müssen in Anbetracht des retrospektiven Studiendesigns und der damit unausweichlich verbundenen Limitationen interpretiert werden. Daraus resultiert ebenfalls die limitierte Patientenanzahl, die vergleichbaren Studien zu diesem Thema entspricht, jedoch die Analyse einzelner Einflussfaktoren auf die betrachteten onkologischen Ergebnisse beeinträchtigt. Ein weiterer Aspekt ist der große Zeitraum der retrospektiven
- Komplikationen, v.a. pharyngokutane Fisteln, sind häufig, jedoch vorwiegend konservativ und selten chirurgisch erfolgreich zu therapieren.

\section{Korrespondenzadresse

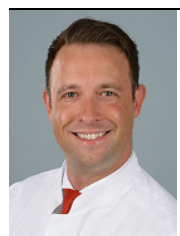 \\ Dr. med. Matti Sievert \\ Hals-Nasen-Ohrenklinik, Kopf- und Hals-Chirurgie, Universitätskliniken Erlangen, Friedrich-Alexander- Universität Erlangen- Nürnberg Waldstraße 1,91054 Erlangen, Deutschland matti.sievert@uk-erlangen.de}

Funding. Open Access funding enabled and organized by Projekt DEAL.

\section{Einhaltung ethischer Richtlinien}

Interessenkonflikt. M. Sievert, M. Goncalves, B. Binder, S.K. Mueller, R. Rupp, M. Koch, S. Dürr, M. Traxdorf, M. Hecht, H. Iro und A.-O. Gostian geben an, dass kein Interessenkonflikt besteht. 
Diese retrospektive Studie erfolgte nach Konsultation der zuständigen Ethikkommission und im Einklang mit nationalem Recht.

Open Access. Dieser Artikel wird unter der Creative Commons Namensnennung 4.0 International Lizenz veröffentlicht, welche die Nutzung, Vervielfältigung, Bearbeitung, Verbreitung und Wiedergabe in jeglichem Medium und Format erlaubt, sofern Sie den/die ursprünglichen Autor(en) und die Quelle ordnungsgemäß nennen, einen Link zur Creative Commons Lizenz beifügen und angeben, ob Änderungen vorgenommen wurden.

Die in diesem Artikel enthaltenen Bilder und sonstiges Drittmaterial unterliegen ebenfalls der genannten Creative Commons Lizenz, sofern sich aus der Abbildungslegende nichts anderes ergibt. Sofern das betreffende Material nicht unter der genannten Creative Commons Lizenz steht und die betreffende Handlung nicht nach gesetzlichen Vorschriften erlaubt ist, ist für die oben aufgeführten Weiterverwendungen des Materials die Einwilligung des jeweiligen Rechteinhabers einzuholen.

Weitere Details zur Lizenz entnehmen Sie bitte der Lizenzinformation auf http://creativecommons.org/ licenses/by/4.0/deed.de.

\section{Literatur}

1. Goodwin WJ Jr. (2000) Salvage surgery for patients with recurrent squamous cell carcinoma of the upper aerodigestive tract: when do the ends justify the means? Laryngoscope 110:1-18

2. Esteller E, Vega MC, López $M$ et al (2011) Salvage surgery after locoregional failure in head and neck carcinoma patients treated with chemoradiotherapy. Eur Arch Otorhinolaryngol 268:295-301

3. Fung K, Teknos TN, Vandenberg CD et al (2007) Prevention of wound complications following salvage laryngectomy using free vascularized tissue. Head Neck 29:425-430

4. Paydarfar JA, Birkmeyer NJ (2006) Complications in head and neck surgery: a meta-analysis of postlaryngectomy pharyngocutaneous fistula. Arch Otolaryngol Head Neck Surg 132:67-72

5. Putten L, Bree R, Doornaert PA et al (2005) Salvage surgery in post-chemoradiation laryngeal and hypopharyngeal carcinoma: outcome and review. Acta Otorhinolaryngol Ital 35:162-172

6. Forster MD, Devlin MJ (2018) Immune checkpoint inhibition in head and neck cancer. Front Oncol 8:310

7. Doescher J, Veit JA, Hoffmann TK (2017) Die 8. Ausgabe der TNM-Klassifikation: Neuerungen für das Fachgebiet Hals-Nasen-Ohren-Heilkunde, Kopf- und Halschirurgie. HNO65:956-961

8. Hoffman HT, Porter K, Karnell LH et al (2006) Laryngeal cancer in the United States: changes in demographics, patterns of care, and survival. Laryngoscope 116:1-13

9. Putten L, Bree R, Kuik DJ et al (2011) Salvage laryngectomy: oncological and functional outcome. Oral Oncol 47:296-301

10. Santoro R, Bini B, Mannelli G et al (2014) Salvage surgery after unsuccessful radiotherapy in early glottic cancer. B-ENT 10:113-120

11. Grün A, Kuhnt T, Schlomm T et al (2020) Repeat radiation for local recurrence of head and neck tumors and in prostate cancer. Dtsch Arztebl Int 117:167-174

12. Ohizumi $Y$, Tamai $Y$, Imamiya $S$ et al (2002) Complications following re-irradiation for head and neck cancer. Am JOtolaryngol 23:215-221

13. Kowalski LP, Carvalho AL (2000) Natural history of untreated head and neck cancer. Eur J Cancer 36:1032-1037

14. Dirven R, Swinson BD, Gao K et al (2009) The assessment of pharyngocutaneous fistula rate in patients treated primarily with definitive radiotherapy followed by salvage surgery of the larynx and hypopharynx. Laryngoscope 119:1691-1695

15. Patel UA, Moore BA, Wax M et al (2013) Impact of pharyngeal closure technique on fistula after salvage laryngectomy. JAMA Otolaryngol Head Neck Surg 139:1156-1162

16. Hausmann J, Kubesch A, Goettlich CM et al (2020) Quality of life of patients with head and neck cancer after prophylactic percutaneous-gastrostomy. Eur JClin Nutr 74:565-572

17. Mekhail TM, Adelstein DJ, Rybicki LA et al (2001) Enteral nutrition during the treatment of head and neck carcinoma: Is a percutaneous endoscopic gastrostomy tube preferable to a nasogastric tube? Cancer 91:1785-1790

18. Holsinger FC, Funk E, Roberts DB et al (2006) Conservation laryngeal surgery versus total laryngectomy for radiation failure in laryngeal cancer. Head Neck 28:779-784

19. Zbären P, Nuyens M, Curschmann J et al (2007) Histologic characteristics and tumor spread of recurrent glottic carcinoma: analysis on wholeorgan sections and comparison with tumor spread of primary glottic carcinomas. Head Neck29:26-32

\section{HNO-Veranstaltungen}

Liebe Leserinnen und Leser, Informationen zur nächsten Jahrestagung der DGHNO-KHC finden Sie hier: https://www.hno.org/2022/index.html

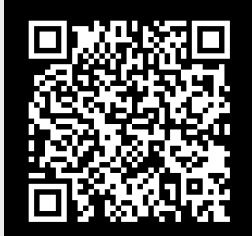

Weitere Veranstaltungen finden Sie unter: https://www.hno.org/ veranstaltungen/index.htm

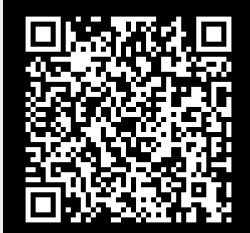

